\title{
Les bases de l'hydraulique pastorale dans le Soudan oriental (Cercle de Gao)
}

\author{
par O. BREMAUD et H. RADIER
}

\section{A. - LE RELIEF \\ LES RÉGIONS NATURELLES}

Le Cercle de Gao est caractérisé par l'extension d'un relief tabulaire d'une grande monotonie, d'où émergent le massif de l'Adrar des Iforas et les illots granitiques du Timétrine.

La pente générale très faible de ces plateaux rayonne autour de l'Adrar des Iforas, vers l'ouest, le sud et le sud-est. Ce pendage, ainsi que la nature des terrains, ont fait apparaître deux falaises concentriques que l'on peut suivre sur le pourtour de l'Adrar des Iforas, à une distance qui varie de $50 \mathrm{~km}$ à l'ouest jusqu'à $150 \mathrm{~km}$ au sud-est.

Falaises et buttes-témoins réalisent ainsi un paysage très comparable à celui des « côtes " de l'est du Bassin parisien. Les falaises sont largement ouvertes par les profondes vallées fossiles qui ont creusé les plateaux en suivant la pente générale, c'est-à-dire vers le Nigcr.

Les lignes simples de ce relief sont fréquemment noyées dans des placages dunaires d'importance variable.

Les massifs de l'Adrar des Iforas, qui se dressent au nord du Cercle, s'inscrivent à l'intérieur d'un triangle équilatéral de 250 à $300 \mathrm{~km}$ de côté, se raccordant par son angle nord-est au Hoggar.

L'aspect physique de l'Adrar des Iforas est très particulier : il apparaît comme un massif montagneux noyé dans ses éboulis jusque mi-hauteur. Ainsi, les anciens points culminants sont devenus des massifs isolés les uns des autres par de larges vallées ensablées ou par de vastes plaines caillouteuses (regs), comme des flots rocheux dans la mer.

Ceci résulte de l'érosion en climat désertique : les orages rares et isolés démantèlent brutalement les sommets et les hautes vallées, mais l'eau des torrents se perd et s'évapore, abandonnant les matériaux entraînés, bien avant d'avoir atteint les plaines et, a fortiori, la mer.

Les massifs les plus importants correspondent à de vastes dômes granitiques rouges, où l'érosion " en boules » ou « en dos d'éléphants » apparaît de façon caractéristique, créant des chaos les plus étranges.

Des massifs de moindre importance correspondent à des intrusions volcaniques dont les roches noires et tranchantes, donnent un aspect sinistre.

Enfin, d'importants bancs de quartzites ou de cipolins, orientés nord-sud, donnent naissance à des crêtes rectilignes en voie d'ensablement.

Au nord de l'Adrar, commence l'immense plaine du Tanezrouft.

Le Timétrine correspond aux contreforts occidentaux de l'Adrar des Iforas. Mais là, les dômes granitiques peu élevés ont été recouverts par des dépôts marins et continentaux. L'érosion récente, burinant ces placages, fait apparaitre un relief saisissant : les dômes granitiques aux parois abruptes, largement dégagés, sont ceinturés de profondes vallées qui s'insinuent en canyons vers l'aval, dans l'épaisseur dos plateaux.

A l'ouest du Timétrine, une vaste zone de dunes s'étend jusqu'en bordure du Bassin de Taoudéni.

La Vallée morte du Tilemsi qui prend sa source entre l'Adrar des Iforas et le Timétrine, et se poursuit jusqu'au Niger qu'elle rejoint à Gao, constitue une région naturelle. En effet, elle drainie la majeure partie des eaux du Timétrine et de l'Adrar en couvrant une vaste région où le relief tabulaire peu ensablé apparaît nettement creusé et morcelé.

L'Azaouad (ou Haoussa de Bourem) est la région qui couvre la rive gauche du Niger à l'ouest du Tilemsi et au sud-ouest du Timétrine. L'Azaouad est couvert de cordons dunaires parallèles orientés sud-ouest-nord-est, que l'on peut suivre sur plus de $100 \mathrm{~km}$ dans la région septentrionale; ces cordons sont plus irréguliers au sud.

Le Bassin oriental du Niger (Haoussa de Gao, d'Ansongo, de Ménaka), englobe les bassins de tous les anciens affluents rive gauche du Niger, au sud et à l'est du Tilemsi. Cette vaste région naturelle déborde largement à l'est et au sud les frontières du Cercle. Il s'agit d'un immense bassin s'étendant sur $800 \mathrm{~km}$ d'ouest en est $(400 \mathrm{~km}$ seulement sur le territoire de Gao); la pente générale 


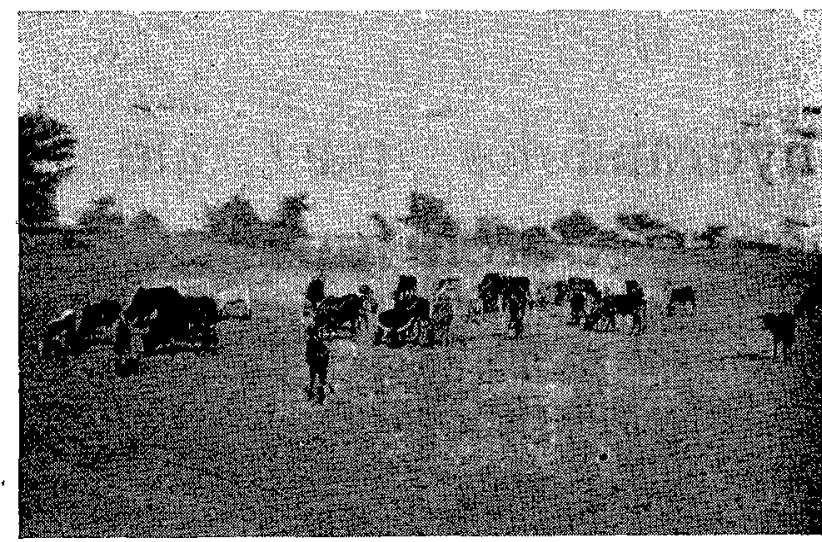

Pâturage aquatique (berges du Niger) pendant la saison sèche.

faible est dirigée vers le sud ou le sud-ouest. Le plateau est ici très largement ensablé; les terrains ne sont souvent visibles qu'en coupe, en bordure des vallées.

Le Gourma est la région situeée sur la rive droite, à l'intérieur de la boucle du Niger. Il est formé de terrains métamorphiques très anciens (schistes et quartzites); les couches, souvent redressées, donnent des lignes de crêtes ou de collines, orientées sud-est-nord-ouest. Les dunes forment localement des trainées discontinues, sauf en bordure du Niger où elles dressent une barrière chaotique de 20 à $30 \mathrm{~km}$ de large.

\section{B. - SYSTÈME HYDROGRAPHIQUE}

\section{a) Le Niger.}

Seul cours d'eau permanent du Cercle, le Niger parcourt $450 \mathrm{~km}$ à l'intérieur du territoire de Gao, y découpant deux parties d'importance très inégale: le Haoussa (rive gauche), le Courma (rive droite).

Aux hautes eaux sa largeur varie de $10 \mathrm{~km}$ dans les plaines inondées à $300 \mathrm{~m}$ au seuil de Tahoussa.'

En franchissant la frontière occidentale du Cercle à Kermachoué, le fleuve offre une large zone d'inondation, encadrée de hautes dunes vives, qui se rétrécit brusquement à la hauteur de Tahoussa.

A cet endroit, divisé en deux bras dont le moins profond s'assèche à la décruc, encaissó entrc scs berges abruptes, il se taille un passage entre les massifs de l'Adrar et du Gourma.

Au sortir de ce seuil, ses eaux s'étalent à nouveau dans une large vallée limitée au nord par des falaises de grès argileux, et au sud par des dunes. A Bourem, il quitte sa direction ouest-est pour piquer sud-est. Vers Ansongo, les falaises s'écartent et le fleuve coule dans une plaine de granit, où il s'enfonce à peine et s'éparpille en de nombreux bras encombrés de rochers.

A la sortie du Cercle, aux environs de Labezzenga, des couches de grès perpendiculaires au cours provoquent des rapides qui interdisent toute navigation de tonnage moyen.

Pendant tout son cours à l'intérieur du Cercle, le Niger ne reçoit aucun affluent permanent. Après chaque tornade, quelques vallées mortes et quelques petits oueds drainent vers son cours les eaux de ruissellement.

La période des hautes eaux se situe entre août et mars. La crue atteint son maximum à Gao en novembre et décembre; le niveau le plus bas apparait en mai-juin; la différence de niveau entre novembre el mai varie de 3 à $5 \mathrm{~m}$. Ces mouvements se trouvent décalés d'un mois environ par $250 \mathrm{~km}$ de parcours.

Aux hautes eaux, dans la partie de son cours située en amont de Tahoussa, les zones d'inondation, berges et îles, se couvrent de prairies aquatiques; après Tahoussa; ces pâturages sont réduits à de petites bandes et même à certains endroits, font totalement défaut.

En saison sèche, le Niger apparaît comme une succession de petits marigors paressant entre des rives d'argile limoneuse noire ou se glissant à travers d'immenses bancs de sable; chaque marigot, large au plus de $400 \mathrm{~m}$, communique avec le suivant par un mince filet d'eau coupé de rapides qui permettent la traversée à gué.

\section{b) Les affluents du Niger.}

Ils constituent un réseau de vallées mortes. Certaines de ces vallées forment de grands espaces dans lesquels l'eau séjourne et 's'évapore : quelquesunes au contraire, présentent pendant l'hivernage un cours torrentiel; d'autres enfin, offrent l'aspect intermédiaire avec des portions torrentielles terminées par des mares. Quelquefois le profil est si indécis que l'eau coule dans les deux sens.

Au nombre de six, les grandes vallées du Haoussa sont toutes situées à l'est du méridien 0 ; ce sont : le Tilemsi, l'Idelimane, l'Ezgueret, l'Assakaraï, l'Azaouak, l'nzahr.

Prenant leurs "sources » soit sur les contreforts de l'Adrar des Iforas, soit dans le Hoggar, ces vallées fossiles offrent plusieurs particularités communes : leur orientation nord-sud, leur régime et leur confluence nigérienne.

Leur partie septentrionale ne s'anime qu'exceptionnellement alors que leurs tronçons méridionaux charrient les eaux des tornades jusqu'aux obstacles 
naturels, seuils ou dunes dont leur lit est encombré.

Une très grande partie de l'eau collectée par les hassins de ces vallées est absorbée par le sol. Bien que leur lit soit foré de nombreux puisards on ne peut parler de cours souterrains. L'eau souterraine d'origine alluviale est presque toujours enfermée dans des poches aux limites très précises.

Les vallées secondaires et les affuents des principales vallées qui présentent un intérêt, soit par leurs ressources hydrologiques exploitées, soit par les possibilités qu'elles offrent sont porlées au tableau suivant :

\begin{tabular}{|c|c|c|}
\hline BASSINS & NOMS DES VALLEES & OBSERVATIONS \\
\hline $\begin{array}{c}\text { Bassin } \\
\text { du } \\
\text { Tilemsi }\end{array}$ & $\begin{array}{l}\text { Irarar. } \\
\text { Tarlit. } \\
\text { Oued el Ouig. } \\
\text { Insatafan. } \\
\text { Oued Tagmart. } \\
\text { Ibdakane. } \\
\text { Iracher Sadidène. } \\
\text { Eguerir. } \\
\text { Ouan Amacine. } \\
\text { Ganchirane. } \\
\text { In Chaouag. } \\
\text { Iracher. } \\
\text { Sadidène. }\end{array}$ & $\begin{array}{c}\text { Possibilités } \\
\text { de } \\
\text { barrages. }\end{array}$ \\
\hline $\begin{array}{c}\text { Bassin } \\
\text { de } \\
\text { l'Idelimane }\end{array}$ & $\begin{array}{l}\text { Adaran Araret. } \\
\text { Agarnadamouss. } \\
\text { Adaran Takelit. } \\
\text { Oued Eranga. } \\
\text { Oued Soukna- } \\
\text { gader }\end{array}$ & $\begin{array}{c}\text { Possibilités } \\
\text { de } \\
\text { barrages. }\end{array}$ \\
\hline $\begin{array}{l}\text { Bassin } \\
\text { de } \\
\text { l'Ezgueret }\end{array}$ & $\begin{array}{l}\text { Oued Intadeini. } \\
\text { Oued Intedouf. } \\
\text { Tigelali. } \\
\text { Tin Amassine. }\end{array}$ & $\begin{array}{c}\text { Possibilités } \\
\text { de } \\
\text { barrages. }\end{array}$ \\
\hline $\begin{array}{c}\text { Bassin } \\
\text { de } \\
\text { l'Azaouak }\end{array}$ & Oued Tadriant. & \\
\hline $\begin{array}{c}\text { Affluents } \\
\text { du } \\
\text { Niger }\end{array}$ & $\begin{array}{l}\text { Andernemel et } \\
\text { son bassin. } \\
\text { Majibo. } \\
\text { Rabarat. } \\
\text { Tin Dereouel. } \\
\text { Adouf. }\end{array}$ & $\begin{array}{c}\text { Possibilités } \\
\text { de } \\
\text { barrages. }\end{array}$ \\
\hline
\end{tabular}

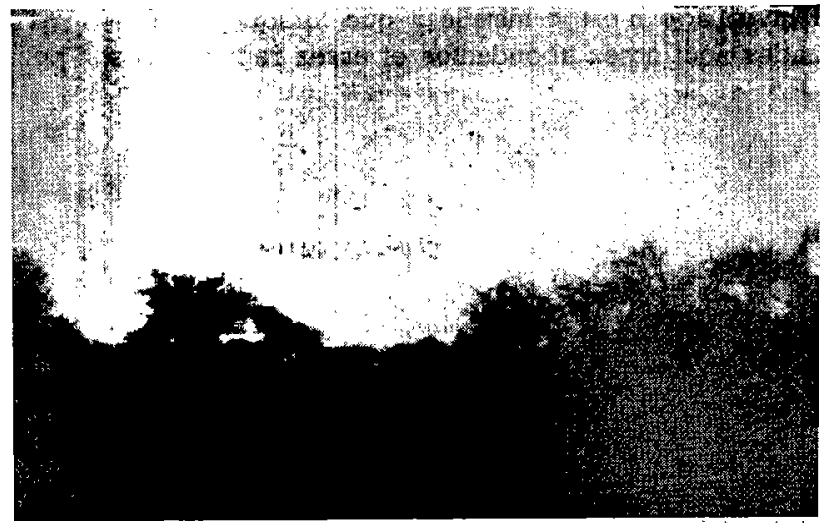

Le lit d'une rivière en saison sèche : toute trace d'eau a disparu.

\section{c) Les vallées du Gourma.}

Le réseau de ces vallées, guidé par l'alternance des couches de schistes et de quartzites, est orienté sud-est-nord-ouest, de façon constante. Certaines vallées se terminent par des bassins fermés; d'autres, obstruées par des traînées de sablo, offrent un chapelet de mares tout au long de leur cours.

Trois vallées prenant leur source au nord de Doro, échappent à cette règle et atteignent le' Niger entre Rharous et Bamba, dans l'ancien bassin d'épandage nigérien.

\section{C. - LE CLIMAT}

Le climat du Cercle de Gao est uniformément qualifié de sahélien, cependant on enregistre certaines différences suivant la latitude.

A l'extrême nord (lat. $22^{\circ}$ ), le climat est nettement saharien, au sud (lat. 150) subsoudanais, au centre (lat. 18 ${ }^{\circ}$ ) sahélien. Dans l'ensemble, c'est un climat sec et chaud, offrant de gros écarts do température (mai : minimum 1909, maximum 4608; janvier : minimum 9o, maximum 37\%3). Les températures nocturnes sont nettement inferieures aux températures diurnes (mai : nocturne, $26^{\circ}$, diurne $43^{\circ}$ ).

Les pluies, dont l'importance varie en raison inverse du degré de latitude, sont en règle générale peu abondantes et irrégulièrement répartios dans le temps et dans l'espace. Cette irrégularité permet d'affirmer que toutes les années sont exceptionnelles. Toutefois, après quelques 'années, on remarque un certain rythme.

La saison des pluies est comprise entre le 15 juillet et le 31 août; cependant, quelques averses la précèdent et la suivent (mai, juin et septembre). 
L'hivernage n'est « installé » que lorsque les tornades sont assez abondantes et assez rapprochées

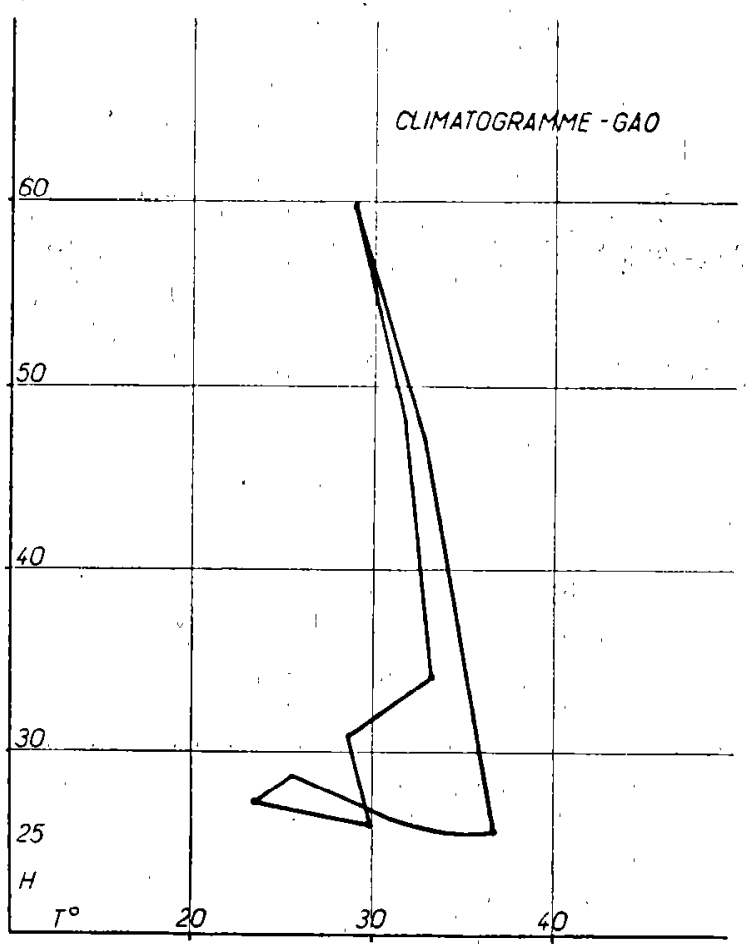

Fig 1. - Climatogramme de la région de Gao.

pour permettre le cycle végétatif du tapis herbacé.

Sauf quelques exceptions, les tornades sont amenées par les vents d'est.
Presque tous les ans, on enregistre une ou deux petites pluies d'hiver.

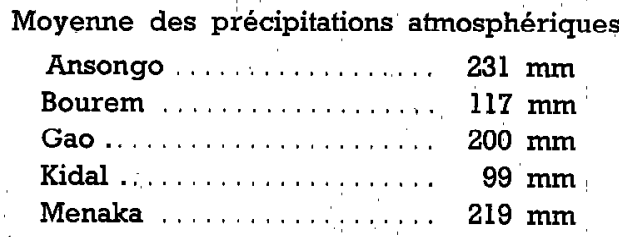

Les moyennes seules donnent une approximation acceptable car, pendant un même hivernage, la lame d'eaú peut être importante sur. le poste météo et nulle à $10 \mathrm{~km}$ du pluviomètre; l'exemple suivant illustre cette remarque :

En 1949, le pluviomètre d'Ansongo indiquait une chute de pluie totale de $149 \mathrm{~mm}$ alors qu'à $10 \mathrm{~km}$ à l'est dans la région comprise entre Asor et Tin Tafarat le total des "précipitations atmosphériques n'a pas atteint $8 \mathrm{~mm}$.

Pour qu'un' hivernage mérite le qualificatif de «bon » il faut :

$1^{\circ}$ que les précipitations soient suffisantes pour que l'eau de ruissellement remplisse les mares:

$2^{\circ}$ que les pluies régulièrement réparties arrosent la totalité du territoire:

$3^{\circ}$ que les jours de pluies se suivent à intervalles assez rapprochés, tous les huit jours environ.

Pour que l'hivernage soit excellent, il est nécessaire que, les trois conditions précédemment énumérées étant remplies, de très fortes tornades viennent clore la saison des pluies et inondent à nouveau tous les bas-fonds.

Le taux d'évaporation est estimé à 90 litresseconde, par kilomètre carré.

Pluies 1949

\begin{tabular}{|c|c|c|c|c|c|c|c|c|c|c|c|c|c|c|}
\hline & & J & $\mathrm{F}$ & M & A & $\mathrm{M}$ & $\mathrm{J}$ & J & A: & SON D & $\begin{array}{l}\text { TOTAL } \\
1949\end{array}$ & 1948 & MOYEWW & ÉCART \\
\hline Ansongo..... & $\begin{array}{l}Q \\
N\end{array}$ & & $\begin{array}{l}2 \\
1 .\end{array}$ & & & $\begin{array}{r}12 \\
3\end{array}$ & $\begin{array}{r}12 \\
2\end{array}$ & $\begin{array}{r}14 \\
3\end{array}$ & $\begin{array}{r}147 \\
9\end{array}$ & & $\begin{array}{r}187 \\
18\end{array}$ & & 271 & $69 \%$ \\
\hline Bourem...... & $Q$ & & & & & 8 & 12 & 22 & 15 & & 58 & 73 & 117 & $50 \%$ \\
\hline Gao......... & $\begin{array}{l}Q \\
N\end{array}$ & & & & $\begin{array}{l}4,4 \\
1\end{array}$ & . & & $\begin{array}{r}54 \\
8\end{array}$ & $\begin{array}{r}43 \\
8\end{array}$ & & $\begin{array}{l}97 \\
17\end{array}$ & 134 & 200 & \\
\hline Kidal...$\ldots \ldots$ & $\begin{array}{l}Q \\
N\end{array}$ & & & $\begin{array}{l}1,5 \\
1\end{array}$ & & $\begin{array}{l}2 \\
1\end{array}$ & $\begin{array}{r}14 \\
3\end{array}$ & & $\begin{array}{r}39 \\
5\end{array}$ & & $\begin{array}{l}58 \\
10\end{array}$ & $\begin{array}{l}82 \\
17\end{array}$ & 99 & $59 \%$ \\
\hline Menaka...... & $\begin{array}{l}\mathrm{Q} \\
\mathrm{N}\end{array}$ & $\begin{array}{l}3 \\
1\end{array}$ & & & & $\begin{array}{l}3 \\
1\end{array}$ & & $\begin{array}{r}23 \\
5\end{array}$ & $\begin{array}{l}78 \\
12\end{array}$ & & $\begin{array}{r}108 \\
19\end{array}$ & $\begin{array}{r}204 \\
30\end{array}$ & 219 & 49. $\%$ \\
\hline
\end{tabular}


Pluies 1931-1940

(Latitude : 16056 nord - Longitude 0020 ouest)

\begin{tabular}{|c|c|c|c|c|c|c|c|c|c|c|c|}
\hline & \multirow{2}{*}{ PLUIE } & \multicolumn{2}{|c|}{$\begin{array}{l}\text { MAXIMUM } \\
\text { en } 24 \text { heures }\end{array}$} & \multicolumn{7}{|c|}{ NOMBRE DE JOURS DE PLUIE SUPÉRJEUR } & \multirow{2}{*}{$\begin{array}{l}\text { NOMBRE } \\
\text { d'années }\end{array}$} \\
\hline & & Valeur & Date & 0,1 & 1 & 10 & 50 & 100 & 150 & 200 & \\
\hline Janvier..... & 0.2 & 1,8 & $21-35$ & 0,1 & 0,1 & 0 & 0 & 0 & 0 & 0 & 9 \\
\hline Février..... & 0,1 & 0,2 & $15-33$ & 0,1 & 0 & 0 & 0 & 0 & 0 & 0 & 9 \\
\hline Mars .. & 0,7 & 7,0 & $26-35$ & 0,1 & 0,1 & 0 & 0 & 0 & 0 & 0 & 10 \\
\hline Avril....... & 0.7 & 5,4 & $25-33$ & 0,4 & 0.2 & 0 & 0 & 0 & 0 & 0 & 10 \\
\hline Mai ... & 2,4 & 24,0 & $21-33$ & 0,1 & 0,1 & 0,1 & 0 & 0 & 0 & 0 & 10 \\
\hline Juin. & 19,0 & 62,0 & $18-33$ & 2,3 & 2,2 & 0,3 & 0,1 & 0 & 0 & 0 & 9 \\
\hline Juillet & 49,5 & 72,0 & $16-33$ & 4,9 & 4,6 & 1,4 & 0,1 & 0,1 & 0 & 0 & 10 \\
\hline Août. & 81,8 & 71,0 & $11-33$ & 7,0 & 6,2 & 2,4 & 0,5 & 0 & 0 & 0 & 10 \\
\hline Septembre... & 23,5 & 30,2 & $4-33$ & 7,4 & 3,0 & 0,6 & 0 & 0 & 0 & 0 & 10 \\
\hline Octobre. & 1,4 & 10,5 & $2-33$ & $0, ?$ & 0,2 & 0,1 & 0 & 0 & 0 & 0 & 9 \\
\hline Novembre.. & 0,4 & 3,4 & $21-31$ & 0,1 & 0,1 & 0 & 0 & 0 & 0 & 0 & 9 \\
\hline Décembre. . & 0 & 0,0 & 0 & 0 & 0 & 0 & 0 & 0 & 0 & 0 & 9 \\
\hline ANNÉE. . & 179,7 & 72,0 & $16-7-33$ & 18,7 & 16,8 & 4,9 & 0,7 & 0 & 0 & 0 & \\
\hline
\end{tabular}

Pluies 1931-1940

ANSONGO

(Latitude : 15041 nord - Longitude : 0.25 est)

\begin{tabular}{|c|c|c|c|c|c|c|c|c|c|c|c|}
\hline \multirow[b]{3}{*}{ Janvier. } & \multirow{2}{*}{ PLUIE } & \multicolumn{2}{|c|}{$\begin{array}{l}\text { MAXIMUM } \\
\text { en } 24 \text { heures }\end{array}$} & \multicolumn{7}{|c|}{ NOMBRE DE JOURS DE PLUIE SUPÉRIEUR } & \multirow{2}{*}{$\begin{array}{l}\text { NOMBRE } \\
\text { d'années }\end{array}$} \\
\hline & & Valeux & Date & 0,1 & 1 & 10 & 50 & 100 & 150 & 200 & \\
\hline & 0,2 & 1,6 & $7-33$ & 0,1 & 0,1 & 0,1 & 0 & 0 & 0 & 0 & 10 \\
\hline Février.. & 0 & 0,0 & + & 0,0 & 0 & 0 & 0 & 0 & 0 & 0 & 10 \\
\hline Mars. . & 0,7 & 6,0 & $26-35$ & 0,4 & 0,1 & 0 & 0 & 0 & 0 & 0 & 10 \\
\hline Avril. & 0 & 0,0 & + & 0 & 0 & 0 & 0 & 0 & 0 & 0 & 10 \\
\hline Mai........ & 33,4 & 130,0 & $22-33$ & 1,4 . & 1,0 & 0,7 & 0,2 & 0,1 & 0 & 0 & 10 \\
\hline Juin . . . . . . & 26,1 & 28,0 & $25-36$ & 3,9 & 3,1 & 1,0 & 0 & 0 & 0 & 0 & 10. \\
\hline Juillet . & 73,9 & 49,0 & $20-36$ & 6,0 & 5,8 & 2,5 & 0 & 0 & 0 & 0 & $\theta$ \\
\hline Août........ & 141,0 & 60,0 & $20-37$ & 9,6 & 9,0 & 4,8 & 0,2 & 0 & 0 & 0 & 10 \\
\hline Septembre... & 45,7 & 65,0 & $3-36$ & 4,4 & 4,2 & 1,6 & 0,1 & 0 & 0 & 0 & 9 \\
\hline Octobre...... & 4,7 & 13,5 & $3-36$ & 0,9 & 0,6 & 0,2 & 0 & 0 & 0 & 0 & 10 \\
\hline Novembre.... & 0 & 0,0 & + & 0 & 0 & 0 & 0 & 0 & 0 & 0 & 10 \\
\hline Décembre.... & 0 & 0,0 & + & 0 & 0 & 0 & 0 & 0 & 0 & 0 & 10 \\
\hline ANNEE. & 325,7 & 130,0 & $22-5-33$ & 26,7 & 24,1 & 10,8 & 0,5 & 0,1 & 0 & 0 & \\
\hline
\end{tabular}


Humidité Gao, Soudan

\begin{tabular}{|c|c|c|c|c|c|c|c|c|c|c|}
\hline & \multicolumn{6}{|c|}{ HUMIDITÉ RELATIVE } & \multicolumn{4}{|c|}{ TENSION DE VAPEUR } \\
\hline & U & $\mathrm{Ux}$ & UN & $\begin{array}{c}0800^{\circ} \\
\text { T M G }\end{array}$ & $\begin{array}{c}1300 \\
\text { T M G }\end{array}$ & $\begin{array}{c}1800 \\
\text { T M G }\end{array}$ & $\mathrm{U}$ & $\begin{array}{c}0800 \\
\text { T M G }\end{array}$ & $\begin{array}{c}1300 \\
T \cdot M G\end{array}$ & $\begin{array}{c}1800 \\
\text { T M G }\end{array}$ \\
\hline Janvier. & 27 & 48 & 14 & 30 & 19 & 25 & 7,8 & 6,1 & 7,3 & 8,6 \\
\hline Février & 28 & 54 & 13 & 30 & 18 & 26 & 9,2 & 6,8 & 7,5 & 10,1 \\
\hline Mars ... & 26 & 50 & 13 & 29 & 15 & 24 & 11,1 & 8,7 & 8,5 & 11,3 \\
\hline Avril ............ & 25 & 51 & 11 & 29 & 11 & 21 & 13,1 & 10,9 & 8,1 & 11,9 \\
\hline Mai $\ldots \ldots \ldots \ldots \ldots$ & 25 & 47 & 15 & 30 & 15. & 19 & 15,2 & 14,5 & 11,2 & 13,2 \\
\hline Juin $\ldots, \ldots \ldots \ldots$ & 33 & 56 & 19 & 45 & 23 & 24 & 19,6 & 20,2 & 16,3 & 15,2 \\
\hline Juillet $\ldots \ldots \ldots \ldots \ldots$ & 47 & 73 & 28 & 62 & 34 & 36 & 23,5 & 23,7 & 20,9 & 19,5 \\
\hline Août $\ldots \ldots \ldots \ldots$ & 59 & 84 & 41 & 74 & 47 & 51 & 25,9 & 25,8 & 24,5 & 23,9 \\
\hline Septembre ........ & 48 & 75 & 27 & 63 & 35 & 39 & 23,6 & 24,1 & 20,7 & 20,4 \\
\hline Octobre........... & 33 & 60 & 14 & 43 & 20 & 28 & $\cdot 16,9$ & 16,7 & 13,6 & 16,0 \\
\hline Novembre $\ldots \ldots \ldots$ & 30 & 52 & 15 & 34 & 21 & 29 & 12,3 & 10,4 & 10,6 & 12,7 \\
\hline Décembre......... & 30 & 50 & 15 & 34 & 21 & 31 & 9,4 & 7,8 & 8,7 & 10,9 \\
\hline ANNÉE. & 32,5 & 58,3 & 18,7 & 41,9 & 29,3 & 29,4 & 15,63 & 12,64 & 13,16 & 14,39 \\
\hline
\end{tabular}

Température Gao, Soudan (altitude $269 \mathrm{~m}$ )

\begin{tabular}{|c|c|c|c|c|c|c|c|c|c|c|}
\hline & \multirow[b]{2}{*}{$\mathrm{T}$} & \multirow[b]{2}{*}{$\mathrm{TX}$} & \multirow[b]{2}{*}{$\mathrm{TX}$} & \multirow[b]{2}{*}{$\begin{array}{c}0800 \\
\text { T M G }\end{array}$} & \multirow[b]{2}{*}{$\begin{array}{l}1300 \\
\text { T M G }\end{array}$} & \multirow[b]{2}{*}{$\begin{array}{l}1800 \\
\text { T M G }\end{array}$} & \multicolumn{4}{|c|}{ VALLEUR EXTREEME } \\
\hline & & & & & & & $\begin{array}{c}\text { Minimum } \\
\text { absolu }\end{array}$ & Date & $\begin{array}{c}\text { Maximum } \\
\text { absolu }\end{array}$ & Date \\
\hline Janvier. & 23,60 & 30,7 & 14,7 & 17,2 & 28,8 & 26,6 & 8,0 & $18-26$ & 37,3 & $9-$ \\
\hline Février. & 25,66 & 32,9 & 16,1 & 19,5 & 31,0 & 28,8 & 11,0 & $1-36$ & 40,0 & $26-$ \\
\hline Mars. & 30,22 & 37,3 & 20,7 & 24,1 & 35,7 & 33,3 & 14,0 & $1-36$ & 43,2 & $24-3$ \\
\hline Avril. & 33,80 & 41,4 & 23,1 & 28,3 & 39,6 & 36,6 & 25,3 & $4-38$ & 46,4 & $17-3$ \\
\hline Mai . & 36,50 & 43,3 & 26,5 & 31,9 & 41,6 & 39,2 & 18,4 & $6-39$ & 46,8 & $21-4$ \\
\hline Juin . & 36,20 & 42,3 & 27,6 & 31,6 & 40,6 & 38,9 & 20,8 & $3-39$ & 45,7 & $9-36$ \\
\hline Juillet & 33,00 & 38,7 & 25,6 & 28,6 & 36,8 & 35,3 & 18,8 & $6-38$ & 43,6 & $21-40$ \\
\hline Août. & 30,72 & 35,7 & 24,3 & 27,0 & 33,9 & 32,7 & 20,0 & $15-36$ & $4 i, I$ & $5-38$ \\
\hline Septembre.. & 32,58 & 38,1 & 25,3 & 28,5 & 36,6 & 34,4 & 19,5 & $23-38$ & 43,4 & $10-38$ \\
\hline Octobre. & 33,42 & 40,1 & 20,8 & 28,3 & 38,4 & 35,5 & 20,5 & $26-39$ & 43,1 & $4-40$ \\
\hline Novembre... & 29,60 & 35,5 & 21,8 & 24,4 & 35,0 & 31,4 & 14,2 & $20-39$ & 41,0 . & $4-38$ \\
\hline Décembre... & 24,80 & 31,6 & 16,5 & 19,1 & 30,0 & 27,1 & 10,0 & $31-36$ & 39,0 & $4-40$ \\
\hline ANNÉE. & 30,85 & 37,30 & 22,25 & 25,71 & 35,67 & 33,32 & 9,0 & $18-1-36$ & 46,8 & 3-5-31' \\
\hline
\end{tabular}


Tableau des principaux points d'ean classés par subdivisions

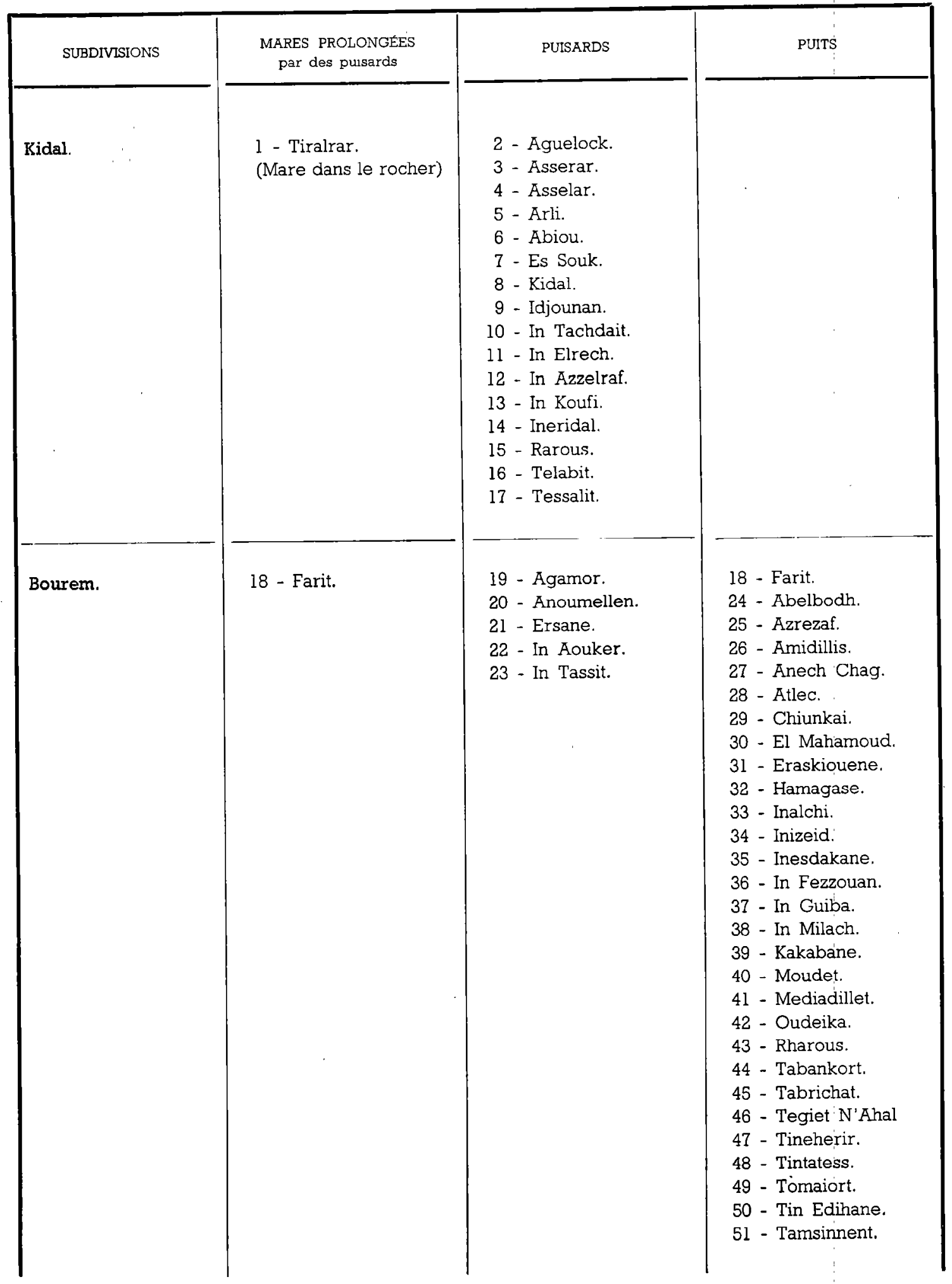


Tableau des principaux points d'eau classés par subdivisions (suite)

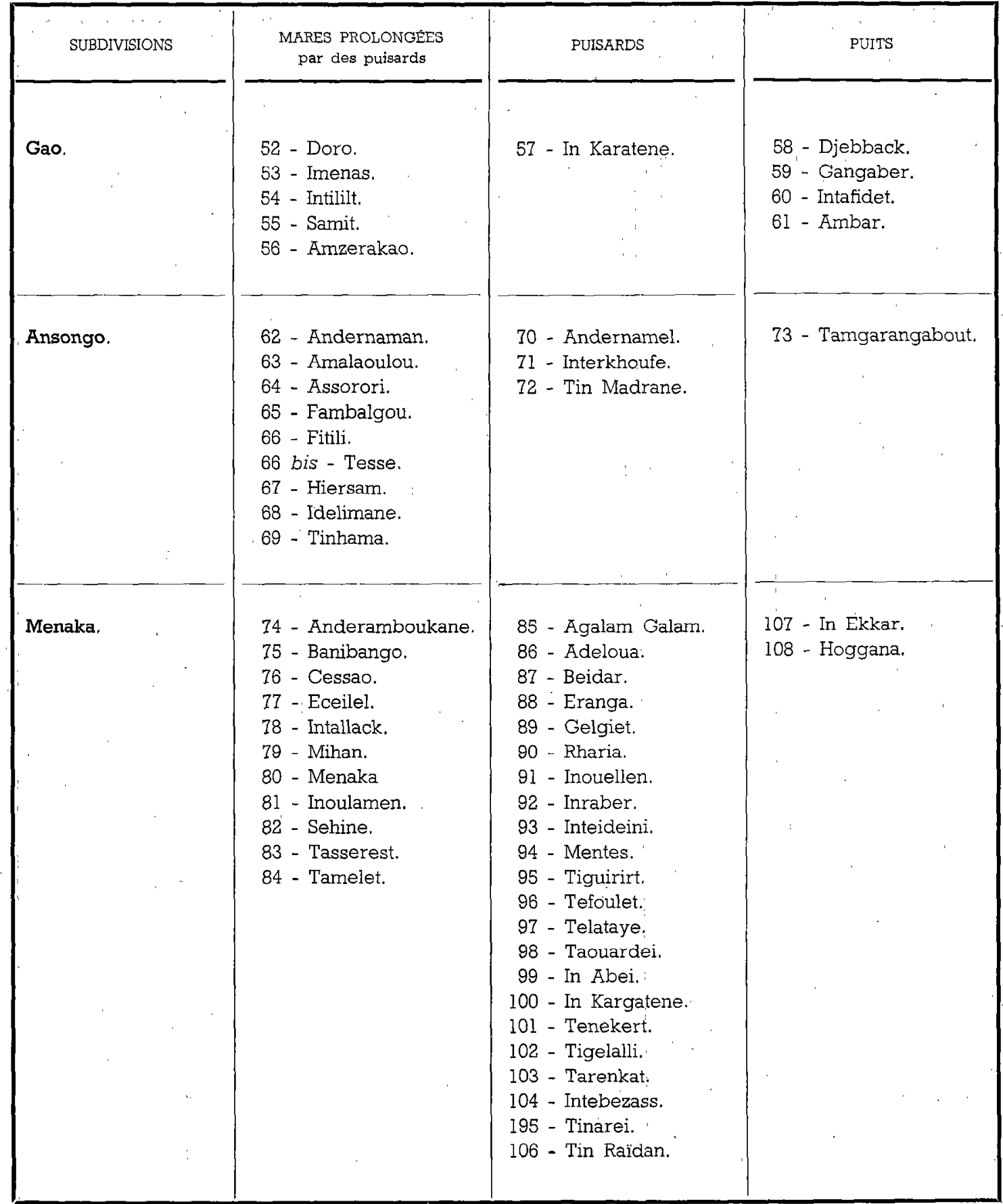

\section{D. - LES POINTS D'EAU}

Pendant la courte période de l'hivernage, le tèrritoire zébré de rivières et de ruisseaux temporaires dans sa partie méridionale et rayé, l'espace d'une journée ou deux, de torrents dans le nord apparaît dans son ensemble constellé de points d'eau, dont l'importance varie de celle de la simple. flaque à celle du lac.

Dès la dernière pluie, toutes les réserves d'eau dormante sous le double effet de l'évaporation et 
de l'infiltration tendent à s'assécher pour donner au pays sa véritable physionomie caractérisée par une extrême sécheresse.

Tiraillé entre le désir de faire paître ses troupeaux et la nécessité de les abreuver, l'homme imprime alors à la vie animale ce rythme pendulaire qui, avec une périodicité variable suivant les espèces, éparpille le cheptel vers les pâturages toujours temporaires, ou des puisards alimentés par les eaux de ruissellement infiltrées.

Sauf quelques unités forées dans des creux de dunes, la majeure partie des systemes de puisards creusés dans le fond des mares ou sur les berges des oueds s'inscrit dans le réseau des vallées.

Les réserves de surface ou souterraines de. nature alluviale présentent un volume limité. Leur

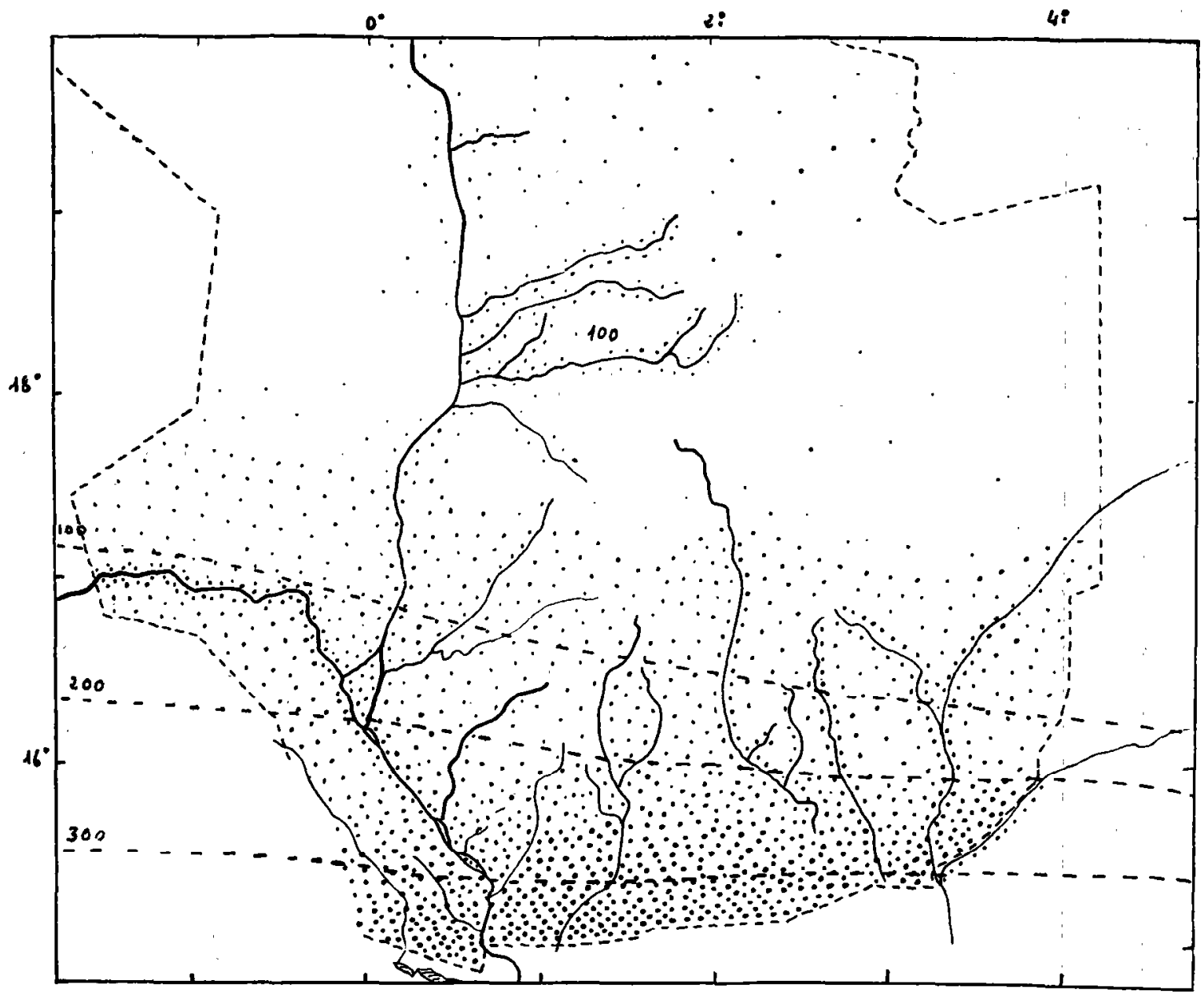

Fig 2. - Carte de la densité des pâturages et des isohyètes.

excentriques pour le rabattre ensuite sur les points d'eau. L'amplitude de ces oscillations s'accroît de mois en mois pour atteindre comme limite la distance que chaque aninal peut parcourir dans le laps de temps séparant les abreuvements que sa physiologie lui impose:-

Excepté les puits profonds de l'Azaouad, les puits de Tabankort, de Tabrichet et de Rarous qui exploitent la nappe du continental intercalaire et les puits récemment forés, tous les points d'eau du Cercle sont formés par des mares presque durce: leur débit, essentiellement variables, dépendent de l'importance des précipitations atmosphériques à laquelle ils sont étroitement liés.

Le nombre de têtes qui abreuvent conditionne également la durée. C'est ainsi que certains groupes de puisards, réputés permanents, ne doivent ce qualificatif qu'au faible nombre des animaux qui les fréquentent, alors que d'autres plus abondants sont asséchés dès la saison froide par de fortes concentrations de betail.

Ces points d'eau permanents de faible puissance, 


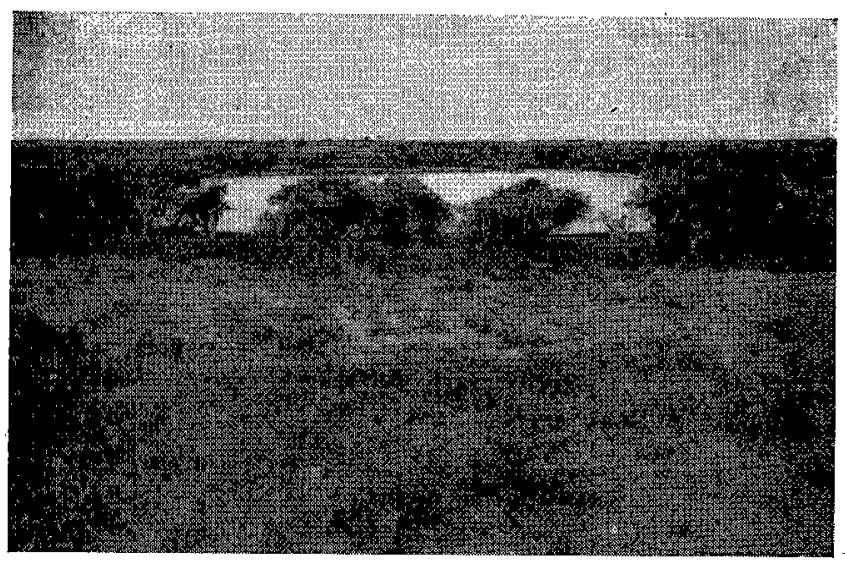

Pâturage de Cenchrus au début de la saison deș pluies (région de Ménaka).

s'ils offrent un intérêt certain comme relais d'élapes ne présentent, en ce qui concerne l'utilisation des parcours, qu'une importance secondaire.

Seuls les points d'eau permanents ou semi-permanents sur lesquels convergent de grands rassemblements ef qui permettent d'utiliser la totalité des pâturages desservis méritent de figurer sur une carte des ressources hydrauliques.

La carte $n^{\circ} 3$ n'a pas la prétention d'être d'une exactitude rigoureuse. Il peut arriver que certaines poches souterraines classées traditionnellement parmi les permanentes s'épuisent alors que, par contre, d'autres étiquetées temporaires prolongent leur durée d'une façon exceptionnelle.

Cette précarité des ressources hydrauliques mérite d'être soulignée. Elle peut entraîner des exodes meurtriers comme ceux de 1949 et 1950 au cours desquels tout le bétail de la vallée de.l'Idélimane dut, en pleine saison sèche, se rabattre à marches forcées sur le fleuve et la vallée de l'Ezgueret.

L'inventaire des ressources hydrauliques et la carte de la répartition du cheptel font apparaître la nécessité de doter le Cercle d'un réseau de puits ou de plans d'eau artificiellement créés plus dense, de façon à décongestionner les parcours de saison sèche et à permettre l'utilisation rationnelle des pâturages.

\section{E. - LES PATURAgES}

(leur distribution, leur charge)

Sur ses 41.000 .000 d'hectares de superficie, le Cercle de Gao compte :

24.014.000 hectares de zone désertique ou saharienne;
8.195.000 hectares de pâturages présahariens;

8.895.000 hectares de pâturages sahéliens. I'isohyete de $100 \mathrm{~mm}$ marque approximativement la limite septentrionale des pâturages sahéliens. Au-dessus commence la zone présaharienne caractérisée par une flore peu variée composée d'espèces très xérothermiques, réduite à une mince bande' parallèle à l'isohyète $100 \mathrm{~mm}$, prolongée d'un appendice superposable à l'Adrar des Iforas et encadré de deux portions sahariennes aux grands espaces désertiques. Dans ces dernières parties de: territoire, à la recherche des pâturages que des tornades localisées font naître et répartissent d'une façon anarchique, seules nomadisent certaines tribus chamelières.

Les pâlurages sahéliens peuvent se distinguer des présahariens par une répartition plus uniforme du couvert végétal, une densité plus forte des flores herbacée et arbustive, l'abondance du Cenchrus qui requiert une certaine pluviosité et la présence de prairies semi-aquatiques qui couvrent les bas-fonds argileux inondés en hivernage.

Sur le trajet du fleuve, le pâturage aquatique, composé de Panicum bourgou, n'offre d'interêt que de mars à juin.

Parmi les nombreuses espèces fourragères qui composent le pâturage de steppe, il convient de ne retenir que celles. dont I'appareil aerien supporte sans être réduit en poussière l'extrême sécheresse et les vents auxquels il est soumis (Panicum, Cenchrus, Aristida; etc.).

Le tapis vegetal de cette région dessine une mosaïque de pâturages de faible hauteur dont les graminées, lorsqu'elles ne sont pas piétinées,

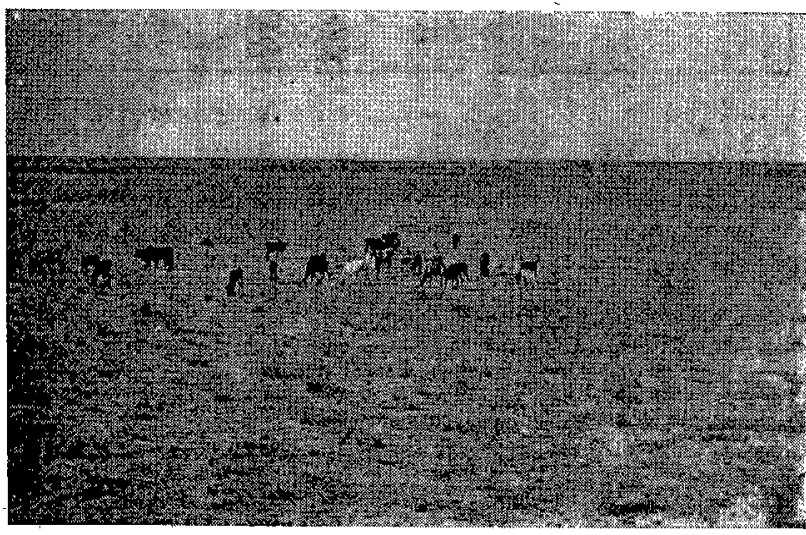

Pâturage en saison sèche dans la région du fleuve. 
restent debout d'un hivernage à l'autre. Aucun de ces pâturages n'est soumis à la pratique du brûlage systématique. Les feux de brousse sont dus à la négligence ou à des causes fortuites.

Compte tenu des espaces nus (regs, montagnes, plaines stériles), de l'irrégularité des prócipitations, etc., le taux de charge à l'hectare est estimé à 0,1 pour la zone sahélienne et 0,025 pour la zone présaharienne.
La capacité de chaque zone s'inscrit comme suit : Zone présaharienne ..... 204.875 unités bétail Zone sahélienne........ 889.500

Capacité totale........ 1.094.375 unités bétail

Le tableau suivant donne les chiffres officiels du recensement du cheotel entretenu sur les pâturages du Cercle, le taux de conversion en unité hétail et $l^{x}$ évaluation en unité bétail.

\begin{tabular}{|c|c|c|c|}
\hline ESPËCE & $\begin{array}{c}\text { NOMBRE EN TÉTES } \\
\text { de bétail }\end{array}$ & $\begin{array}{c}\text { TAUX } \\
\text { de conversion }\end{array}$ & $\begin{array}{l}\text { NOMBRE EN UNITES } \\
\text { Bétail }\end{array}$ \\
\hline Bovins. & 309.298 & $1 / 1$ & $309: 298$ \\
\hline Ovins, Caprins...... & 1.364 .406 & $1 / 10$ & $136: 440$ \\
\hline Chevaux.... & 6.413 & $1 / 1$ & 6.413 \\
\hline Anes... & 52.732 & $1 / 2$ & 26.366 \\
\hline Chameaux... & 49.708 & $1 / 1$ & 49.708 \\
\hline & & TOTAL. . & 528.225 \\
\hline
\end{tabular}

I'étude du dernier tableau amène aux constatations suivantes :

10 dans la bande fluviale surchargée, 82.077 unités sont en surnombre;

$2^{\circ}$ les parcours de la zone présaharienne peuvent entrelenir 94.998 unités de plus;

$3^{\circ}$ les pâturages de l'hinterland sahélien tout en absorbant l'excédent de la région riveraine sont susceptibles de recevoir un complément de 471.152 unités.

Compte tenu de la sous-estimation inhérente aux recensements officiels qui ont servi de base pour les calculs, les chiffres indiquant les possi- bilités de charge des parcours réclament certaines corrections.

L'administration porte sur ses rôles d'impôts tous les animaux, sans distinction d'âge, déclarés par les éleveurs. D'après ses sondages 'et les chiffres de ses vaccinations, le Service de l'Élevage estime que les effectifs recensés représentent les $3 / 5$ du troupeau. Les 2/5 qui échappent au recensement correspondent approximativement à l'effectif des jeunes (bovins, chameaux, chevaux, anes de moins de 3 ans, ovins et caprins de l'année).

Le tableau ci-après donne l'évaluation en unités bétail des jeunes.

\begin{tabular}{|c|c|c|c|}
\hline ESPËCES & $\begin{array}{l}\text { NOMBRE DE TÊTES } \\
\text { de Bótail }\end{array}$ & $\begin{array}{l}\text { TAUX } \\
\text { de conversion }\end{array}$ & $\begin{array}{l}\text { NOMBRE EN UNITÉS } \\
\text { Bétail }\end{array}$ \\
\hline Bovins $\ldots \ldots \ldots \ldots \ldots \ldots$ & 206.198 & $1 / 3$ & 68.732 \\
\hline Ovins et Caprins... & 909.604 & $1 / 20$ & 45.480 \\
\hline Chevaux $\ldots \ldots \ldots \ldots \ldots \ldots \ldots$ & 4.276 & $1 / 3$ & 1.425 \\
\hline Anes. $\ldots \ldots \ldots \ldots \ldots \ldots \ldots \ldots$ & 35.154 & $1 / 6$ & 5.859 \\
\hline Chameaux................. & 33.138 & $1 / 3$ & 11.400 \\
\hline & & TOTAL........ & 132.896 \\
\hline
\end{tabular}


Les terres pastorales du Cercle de Gao apparaissent en mesure d'assurer dans de bonnes conditions, sous réserve que tous les pâturages puissent être ouverts, l'entretien de 1.094 .000 unités bétail de $500 \mathrm{~kg}$, soit 433.000 unités de plus que celles qui composent son élevage actuel.

De la réalisation d'un programme d'hydrau- $2^{\circ}$ un accroissement notable de l'effectif (433.000 unités):

$3^{\circ}$ une amélioration de la qualité; le taux de charge a été établi assez largement, d'une part en fonction des besoins d'une unité bétail de $500 \mathrm{~kg}$, et, d'autre part, compte tenu des. variations de la densité fourragère.

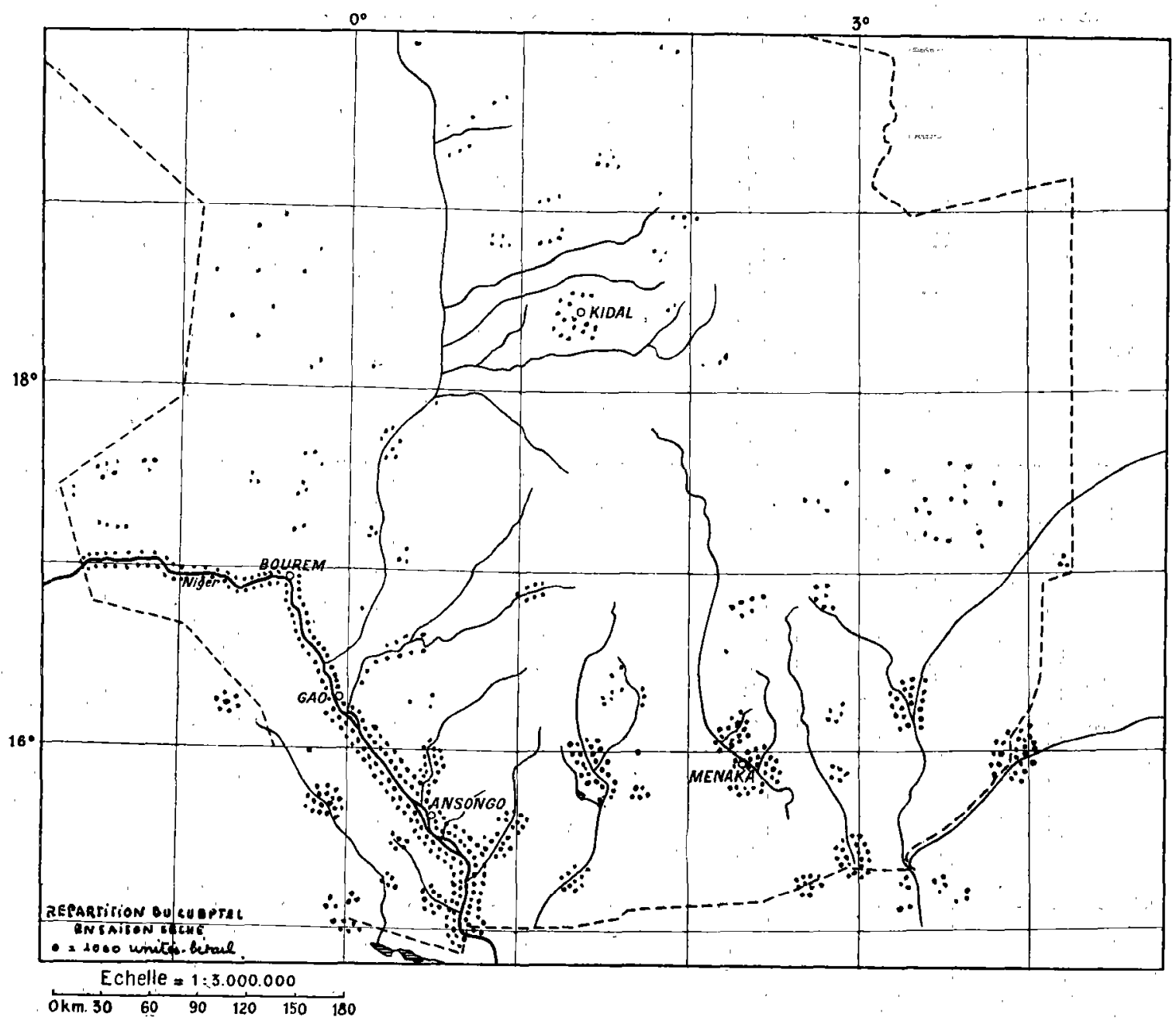

Fig. 3. - Carte do la rópartition du cheptel en saison sèche (chaque point représente 1.000 unités-bétail).

lique pastorale dotant le Cercle d'un réseau suffi'sant de puits ou de plans d'eau artificiels, dependent :

$1^{\circ}$ la décongestion de la zone fluviale et une meilleure répartition dans I'hinterland du bétail actuellement concentré sur des superficies insuffisantes:
De: la mise à la disposition du bétail, pendant toute l'année, d'une quantité de fourrage suffisante, d'un abreuvement meilleur et plus régulier, de la réduction des trajets quotidiens résulteront une augmentation de poids et un accroissement de la production laitière, à laquelle dans une certaine mesure est liée la précocité. 
Effectif du cheptel par espèces, subdivisions et groupements pastoraux

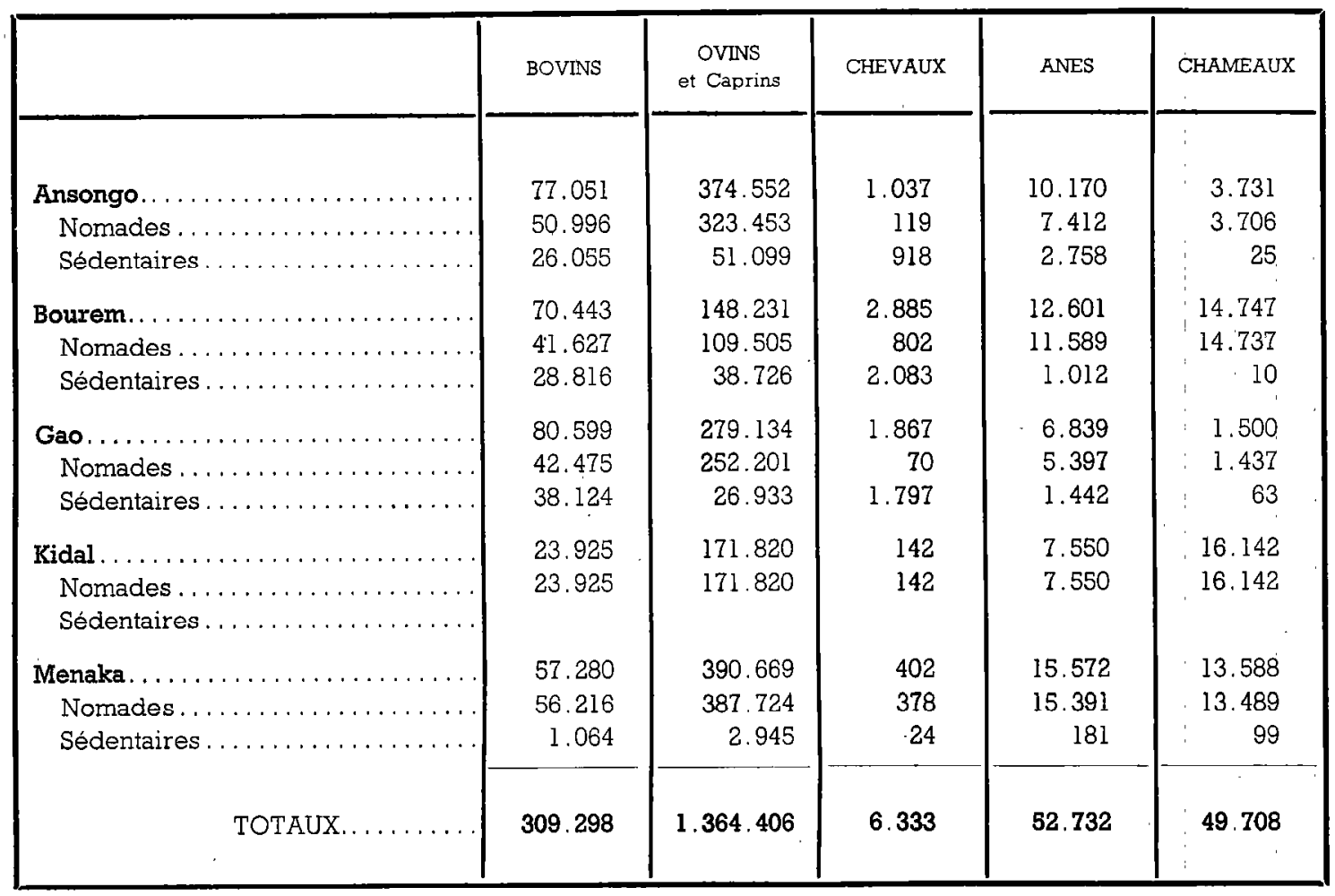

Effectifs du cheptel de la zone présaharienne par subdivisions

\begin{tabular}{|c|c|c|c|c|c|c|}
\hline - & BOVINS & $\begin{array}{l}\text { OVINS } \\
\text { et Caprins }\end{array}$ & CHEVAUX & ANES & CHAMEAUX & $\begin{array}{l}\text { UNITÉS } \\
\text { Bétail }\end{array}$ \\
\hline Ansongo $\ldots \ldots \ldots \ldots \ldots \ldots$ & 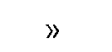 & 》 & $»$ & $»$ & $»$ & $\gg$ \\
\hline Bourem .... & 22.700 & 40.102 & $》$ & 7.227 & (1) 14.737 & 45.060 \\
\hline Gao ......... & 3.319 & 15.046 & 11 & 925 & (1) 328 & 5.625 \\
\hline Kidal. . . . . . . . . . . . & 18.925 & 121.820 & 142 & 6.550 & (1) 16.142 & 50.666 \\
\hline Menaka. . . . . . & $》$ & $»$ & $»$ & 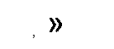 & (1) 8.525 & 8.525 \\
\hline TOTAUX. & 44.944 & 176.968 & 153 & 14.702 & 39.732 & 109.876 \\
\hline
\end{tabular}


Effectifs par subdivisions du cheptel de la zone sahélienne

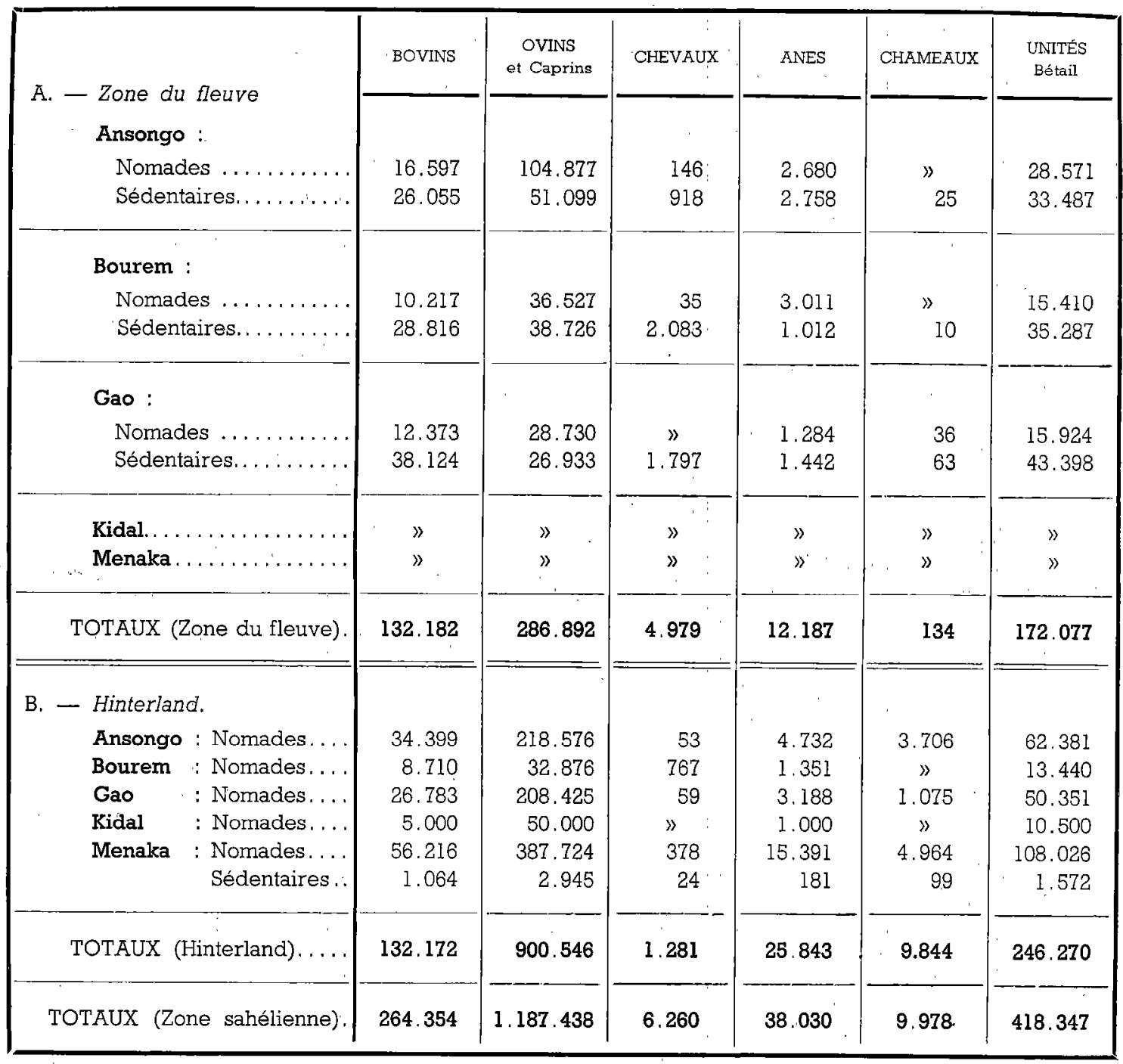

Tableau récapitulatif

Répartition du cheptel dans les diverses zones de pâturages

\begin{tabular}{|c|c|c|c|c|c|c|}
\hline & BOVINS & $\begin{array}{l}\text { OVINS } \\
\text { et Caprins }\end{array}$ & CHEVAUX & ANES & CHAMEAUX & $\begin{array}{l}\text { UNITÉS } \\
\text { Bétail }\end{array}$ \\
\hline Zone présaharienne $\ldots \ldots \ldots$ & 44.944 & 176.968 & 153 & 14.702 & 39.732 & 109.877 \\
\hline Zone sahélienne : & & & & & & \\
\hline Zone du fleuve ... & 142.182 & 286.892 & 4.979 & 12.187 & 134 & 172.077 \\
\hline Hinterland . . . . & 132.172 & 900.546 & 1.281 & 25.843 & 9.842 & 246.271 \\
\hline TOTAUX. & 309.298 & 1.364 .406 & 6.413 & 52.732 & 49.708 & 528.225 \\
\hline
\end{tabular}


Utilisation des pâturages (Possibilités offertes par les pâturages)

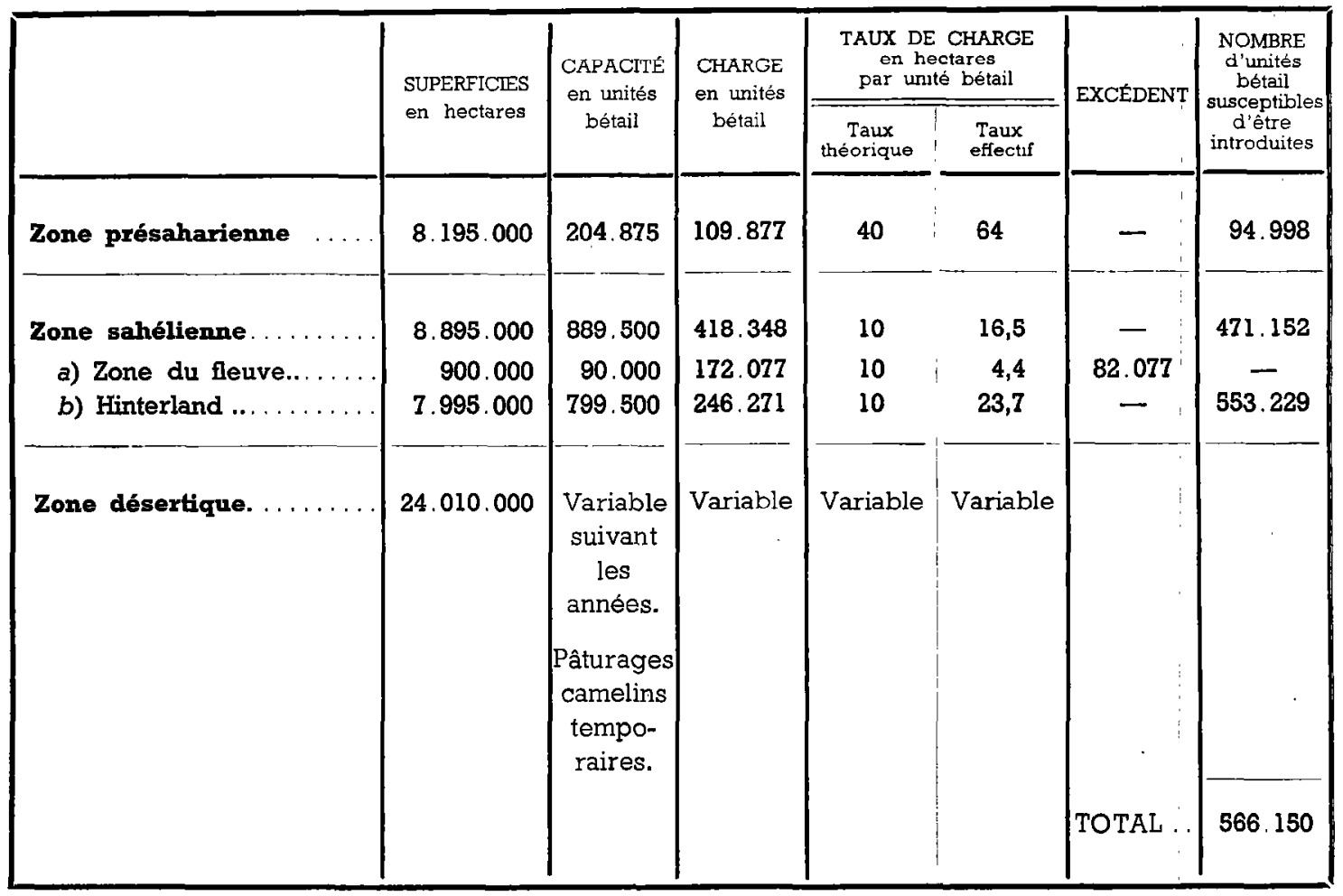

Dans l'état actuel de l'équipement hydraulique sur les 16.090.000 hectares de pâturages, 8.894.000 hectares seulement sont utilisés à fond, 7.196.000 hectares restent inutilisés ou ne sont parcourus que hâtivement pendant l'hivernage et le début de la saison froide (I).

La mise en exploitation de cette superficie nécessite l'ouverture d'environ 100 points d'eau.

\section{PROGREMIMIE D'HYDRAULIQUE PASTORALE}

Avant de jeter les bases d'un programme d'hydraulique, il convient de se pénétrer d'un principe trop souvent oublié : toutes les ressources souterraines connues des pasteurs sont exploitées au maximum.

Il semble donc inutile, sous prétexte d'une modernisation et dans l'espoir d'augmenter le débit, de forer des ouvrages coûteux dans les nappes superficielles. Ces puits, creusés sur les berges des mares pour éviter les détériorations qu'entraînerait leur submersion pendant l'hivernage, pré-

(1) Un point d'eau couvre une superficie de 15 kilomètres de rayon, soit 70.685 hectares. senteraient l'inconvénient d'être plus profonds que les puisards sans offrir un débit supérieur au trou d'homme. D'autre part, alimentés par une nappe de volume limité, leur durée ne saurait dépasser celle des puisards indigènes. De toute façon, par un ou plusieurs ouvrages de ce type on ne peut espérer atteindre la puissance d'abreuvement des multiples puisards qui exploitent les réserves alluviales.

La permanence des points d'eau temporaires actuels ne peut être obtenue qu'à condition de poursuivre le fonçage jusqu'aux nappes profondes.

Le programme doit tendre à équiper les régions laissées vierges ou insuffisamment parcourues.

Ce programme devrait être établi, uniquement en fonction des résultats recherchés, c'est-à-dire sans qu'interviennent, lors de la détermination des lieux d'implantation des points d'eau à créer, des considérations autres que celles se rapportant à l'ouverture de régions pastorales inutilisables par suite du manque d'eau, ou à la meilleure utilisation de pátures périodiquement surchargées.

Une carte provisoire des nappes profondes exploitables a été dressée d'après les observations recueillies au cours des missions de prospection hydro-géologiques et des campagnes d'études de géophysique et les faits nouveaux apportés par les 
iravaux de sondage. La mise à jour de ce document se poursuit; mais déjà, en l'état actuel de nos connaissances, il est possible d'ébaucher un programme de travail.

Sur le socle cristallin (Adrar des Iforas, sa bordure et le Gourma) les ouvrages de retenue d'eau de ruissellement sont seuls à envisager. Trois procédés d'une inégale valeur permettent la rétention des eaux de pluie : les barrages de surface, les barrages souterrains (1), et l'approfondissement des mares.

Les barrages de surface, à condition d'être du type à déversoir latéral sur sol naturel, réclament des travaux de terrassement plus ou moins importants suivant la topographie du lieu d'implantation mais ne nécessitent que peu d'entretien. Par ailleurs, ils offrent l'avantage de retenir un volurne d'eau considérablement supérieur à celui du terrassement que requiert leur construction.

L'inondation qu'ils provoquent favorise le reboisement du bief amont et contribue à créer sur de vastes superficies un micro-climat moins sévère que celui de la brousse environnarte.

Les barrages souterrains ont pour objet la création, dans les vallées à lit perméable, de réserves artificielles d'eaux infiltrées exploitables par puits ou puisards. La majeure partie des nappes alluvionnaires naturelles sont dués à la présence de

(1) Le point d'eau de Djounan dans l'Adrar des Iforas, avec ses trois réserves étagées, est l'exemple naturel duquel on peut s'inspirer pour la construction de ce genre d'ouvrage. bouchons impcrméables obturant le lit souterrain des vallées.:

Le barrage souterrain offre l'avantage d'éviter les pertes par évaporation. Il permet de doter de points d'eau certaines vallées de montagne gravées dans des roches cristallines et tapissées d'un lit de graviers ou de sables interdisant la retenue de l'eau en surface.

L'obligatoire implantation des barrages dans les vallées vives d'hivernage aux endroits où les conditions géographiques et géologiques sont réunies contrarie leur distribution suivant un plan idéal d'utilisation totale des parcours. Cet inconvénient restreint, dans une grande part, les possibilités de baser un programme d'hydraulique sur ce genre de travaux. Leur construction devra en principe êlre linnilée aux régions dépourvues d'eau souterraine.

L'approfondissement des mares naturelles temporaires implique la mise en cuvre d'importants moyens mécaniques. A chaque mètre cube d'eau retenue correspond un égal volume de terre remuée. Cette action n'apparaît rentable que dans les territoires où la densité du réseau de voies de. communications et l'état des pistes permettent l'acheminement de matériel lourd de terrassement sur les points d'utilisation et où la proximité des centres industrialisés garantit le bon entretien d'engins coûteux.

A raison de 301 par unité tous les deux jours, $2.250 \mathrm{~m}^{3}$ d'eau sont nécessaires à l'abreuvement de 500 'unités bétail pendant 10 mois par an.

Théoriquement, une telle réserve nécessite l'aménagement d'une tranchée de $100 \mathrm{~m}$ de long, sur 11,25 $\mathrm{m}$ de large et $2 \mathrm{~m}$ de profondeur.

Superficie desservie par les points d'eau

\begin{tabular}{|c|c|c|c|c|}
\hline \multirow[t]{2}{*}{ SUBDIVISIONS } & \multirow{2}{*}{$\begin{array}{c}\text { NOMBRE } \\
\text { de points d'eau }\end{array}$} & \multirow{2}{*}{$\begin{array}{c}\text { PORTION } \\
\text { de fleuve } \\
\text { évaluée } \\
\text { en kilomètres }\end{array}$} & \multicolumn{2}{|c|}{$\begin{array}{l}\text { SUPERFICIE EN HECTARES } \\
\text { des zones exploitées } \\
\text { à fond commandées par }\end{array}$} \\
\hline & & & les points d'eau & le fleuve \\
\hline Ansongo.... & 12 & 140 & 848.220 & 420.000 \\
\hline Bourem.... & 34 & 175 & 2.403 .290 & 525.000 \\
\hline Gao....... & 10 & 105 & 706.850 & 315.000 \\
\hline$\ldots \ldots \ldots$ & 17 & $\gg$ & 1.201 .645 & $\gg$ \\
\hline Menaka.. & 35 & $»$ & 2.473 .975 & $»$ \\
\hline$\because$ & & TOTAL & 7.633 .980 & 1.260 .000 \\
\hline
\end{tabular}


En pratique, compte tenu de l'évaporation (90 l/seconde par $\mathrm{km}^{2}$, soit $319 \mathrm{~m}^{3}$ pour un plan d'eau de $1.125 \mathrm{~m}^{2}$ ), de l'infiltration et du volant de sécurité destiné à éviter l'abreuvement dans un bourbier en fin de saison sèche, la profondeur doit être portée à $4 \mathrm{~m}$, ce qui représente $4.500 \mathrm{~m}^{3}$ de terrassement (1).

Dans les régions détentrices de ressources hydrauliques souterraines, la préférence sera accordée, on règle générale, aux puits ou aux forages. Quelles considérations permettront de guider le choix entre puits ou forages?

\section{$1^{\circ}$ La profondeur du toit de la nappe}

Dans la région du Tilemsi et dans le Bassin oriental du Niger, dès que l'on s'éloigne des affleurements d'alimentation, les nappes profondes emprisonnées sous des terrains imperméables s'enfoncent régulièrement vers l'ouest.

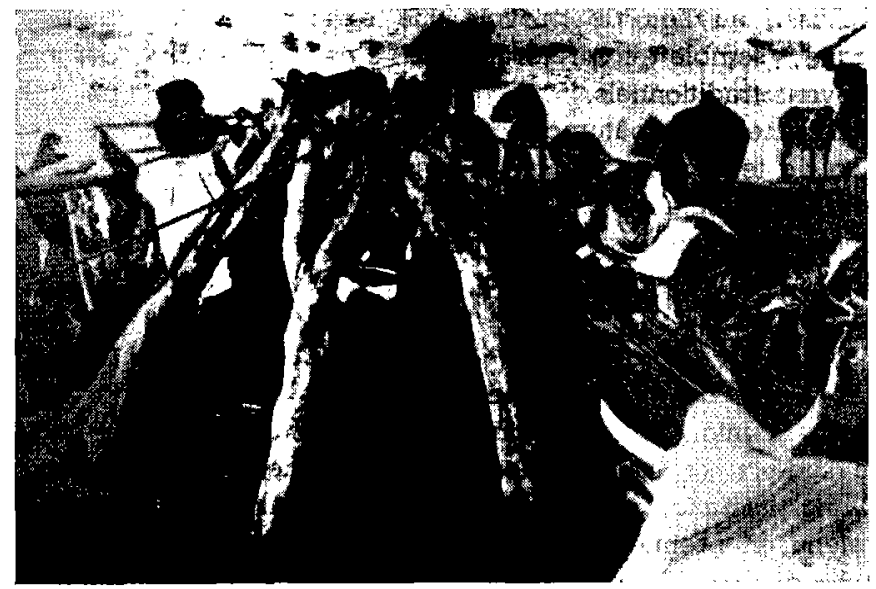

Un puits à Tin Taless.

Remarquer les moyens de puisage employés par les autochtones : poulies en bois montées sur fourches, puisettes en peau, traction animale.

le sud-est. L'épaisseur de terrains stériles à traverser pour atteindre l'eau captive va croissant en direction du sud.

Les services de l'hydraulique estiment que la limite de possibilité de creusement d'un puits (par les méthodes actuelles) se situe vers $80 \mathrm{~m}$ de profondeur.

Par conséquent, Jorsque le toit de la nappe se situera au-delà de cette profondeur, il faudra avoir recours aux forages.

Notons que la notion de nappe captive implique celle d'artésianisme : dès que le plafond de la nappe est percé, celle-ci tend à se mettre en équilibre hydrostatique avec sa surface libre située dans la zone d'alimentation. On obtient donc dans le forage un niveau d'équilibre peu éloigné de la surface. L'installation d'un puits citerne coiffant l'ouvrage permet l'exploitation de la nappe par les méthodes indigènes (2).

(1) Au Sénégal, une excavation de $60.000 \mathrm{~m}^{3}$ $(100 \times 200 \times 3)$, vient d'être mise en eau. Le calcul suivant donne son potentiel d'abreuvement :

- Évaporation $=5.680 \mathrm{~m}^{3} / \mathrm{an}$

- Volume d'eau résiduel en fin de saison impropre à l'abreuvement $=10.000 \mathrm{~m}^{3}$.

- Volume d'eau utilisable $=44.350 \mathrm{~m}^{3}$.

Soit l'abreuvement de 10.000 unités bétail pendant dix mois à raison de 301 tous les deux jours ou 5.000 unités bétail abreuvant quotidiennement.

(2) Deux exemples d'artésianisme :

- le puits de Djebock atteint le toit de la nappe vers $72 \mathrm{~m}$ et le niveau de l'eau s'équilibre vers $55 \mathrm{~m}$;

\section{$2^{\circ}$ L'importance de l'artésianisme.}

L'artésianisme dans un puits présente deux inconvénients :

- il constitue un danger grave pour l'équipe de puisatiers au travail;

- il interdit l'achèvement convenable de la base du puits et, par suite, ouvre la voie aux éboulements.

Par conséquent, dès que l'artésianismé dépassera quelques mètres ( $5 \mathrm{~m}$ au maximum), il deviendra hautement préférable de recourir aux forages.

La proportion et la répartition des puits et des forages restent donc fonction des possibilités hydrogéologiques et ne peuvent être déterminées que compte tenu des enseignements apportés par les études de prospection.

La réalisation d'un important programme d'hydraulique pastorale nécessitera la surveillance attentive des travaux par des géologues qui, interprétant au fur et à mesure les renseignements fournis par chaque ouvrage nouveau, détermineront avec plus de précision les emplacements des ouvrages suivants, en vue d'obtenir les meilleurs rendements.

La position excentrique de la région, l'absence d'infrastructure industrielle, la pénurie de personnel spécialisé, l'hermétisme des populations pastorales au machinisme, ne plaident pas en faveur de la mécanisation des installations de surface.

- le sondage d'In Aoukert atteint vers $140 \mathrm{~m}$ une nappe s'équilibrant à $43 \mathrm{~m}$ seulement du sol. 
Aussi, au lieu de prévoir une exhaure mécanique, semble-t-il préférable de s'en tenir' aux moyens traditionnels.

Les meilleurs pâturages correspondent à la portion de territoire axée sur la ligne Intillit-ÃnsongoMenaka-Tamelet. C'est également sur ces parcours que transhume la majeure partie du cheptel. Les efforts doivent en premier lieu porter sur cette région.

Toutefois, l'équipement des pâturages de l'Azaouad, compte tenu de l'importance du troupeau Kounta, de sa tendance au glissement vers le sud-est, de la présence certaine d'une nappe d'une puissance suffisante, mérite également d'être retenu en première urgence.

La carte no 3 définit les grandes lignes du programme volontairement schématisé; ce canevas devra, dans le détail, subir les modifications que pourra suggérer une étude approfondie des régions a équiper; notamment en ce qui concerne les coordonnées exactes pour l'implantation de chaque ouvrage, lesquelles seront fonction de la densité du couvert végétal, de la structure géologique et du faciès topographique.

- On remarquera l'alignement des premiers travaux sur des axes initiaux à partir desquels s'effectuera l'aménagement des lignes parallèles.

La densité du réseau est calculée sur lạ base d'un point d'eau tous les $30 \mathrm{~km}$. Chaque point desservant une superficie de 70.685 ha devra assurer un débit minimum journalier de $250 \mathrm{~m}^{3}$ en zone sahélienne et de $60 \mathrm{~m}^{3}$ pour la zone présaharienne.

\section{CONCLUSION}

L'équipement hydraulique idéal du Cercle de Gao avec 100 points d'eau dont le prix de revient unitaire peut être estimé à 8 millions de francs, nécessite l'investissement de 800 millions.

Sans tenir compte de la plus-value que prendra le troupeau par suite d'une nourriture et d'un abreuvement plus réguliers, le sirnple accroissement d'effectif (433.000 unités bétail) que l'on peut attendre de l'exécution du programme projeté fait espérer en vingt ans une augmentation du capital bétail évaluée à 4.336 millions.

Par ailleurs, sans prétendre sédentariser les masses pastorales que l'épaisseur de la lame d'eau annuelle et l'irrégularité de sa répartition contraindront encore à des déplacements, la réalisation du programme projeté permettra de réduire sensiblement l'amplitude des mouvements transhumantiels, de fractionner les parcours, d'assurer une meilleure distribution spatiale du, cheptel évitant ainsi la surcharge des pâturages et limitant les risques d'épizooties. 


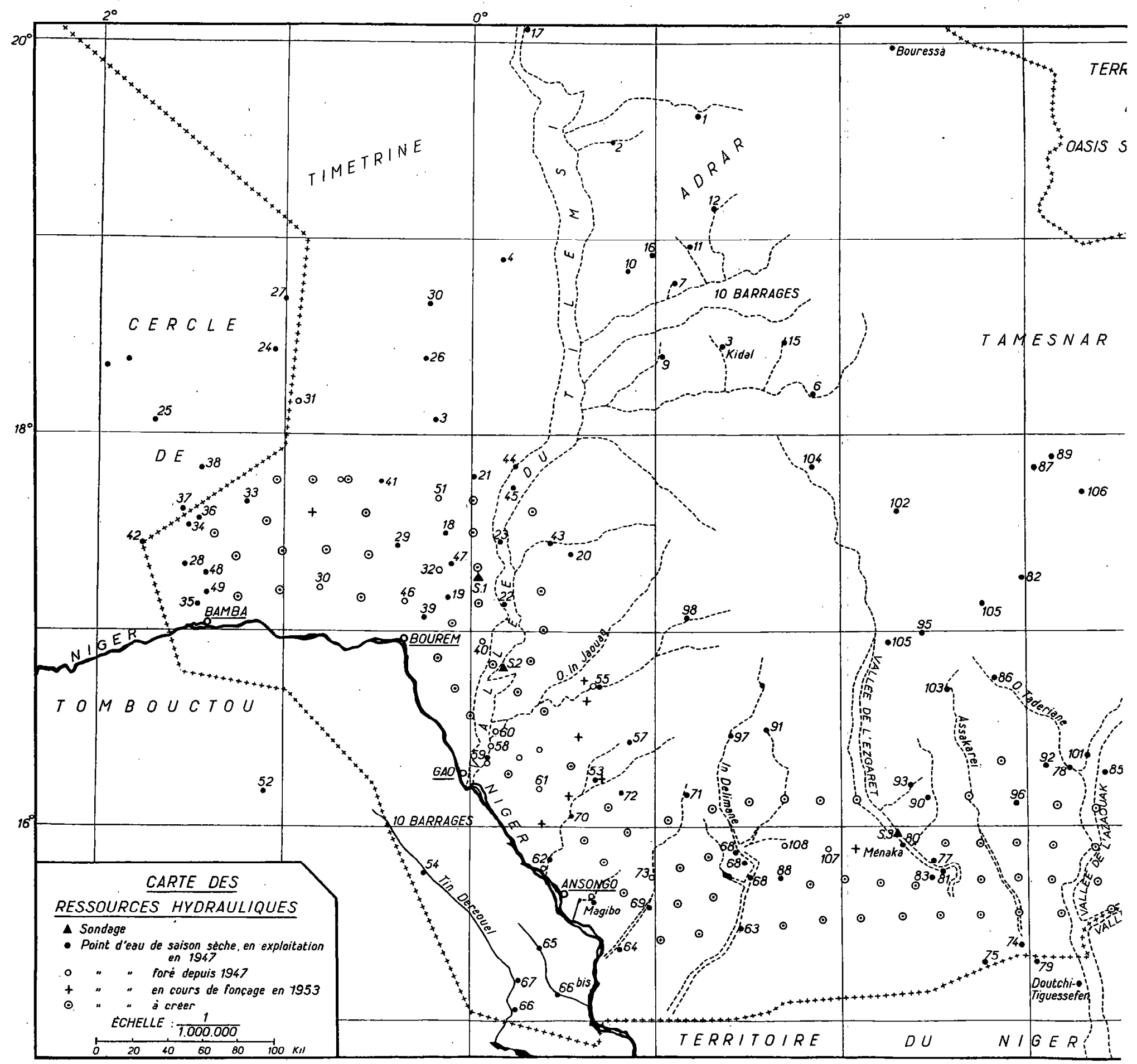




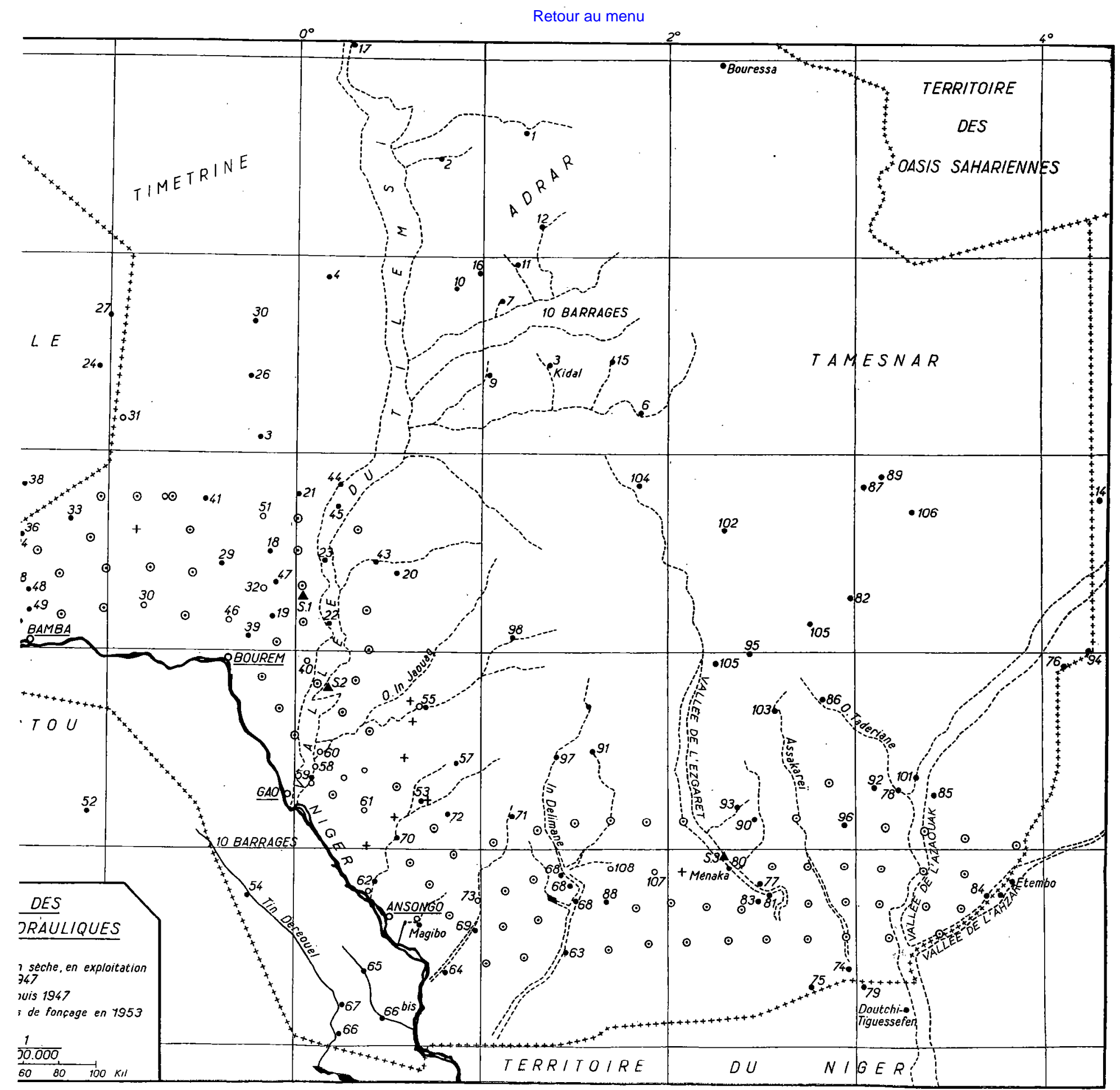

